\title{
Clinical leadership development workshop for licenced practical nurses in supportive living in Alberta: An exploratory qualitative study
}

\author{
Parivash Enghiad, Carol Ewashen, Lorraine Venturato* \\ University of Calgary, Calgary, Alberta, Canada
}

Received: October 9, 2020

DOI: $10.5430 /$ jnep.v11n2p66

\author{
Accepted: October 13, 2020 \\ Online Published: October 25, 2020 \\ URL: https://doi.org/10.5430/jnep.v11n2p66
}

\begin{abstract}
Objective: Since 2013, the scope of practice of LPNs in Alberta, Canada has expanded to include leadership in SL that requires that the development of new CL skills be prioritized. To date, few SL work-based educational programs have been devoted to developing CL skills for LPNs. The objective of this study is the assessment of the impact of a brief patient group education intervention (Conversation Maps ${ }^{T M}$ ) in people with type 2 Diabetes Mellitus.

Methods: An exploratory qualitative design was used, incorporating multiple data collection methods, including individual and focus group interviews, and a demographic questionnaire. Interview data were analyzed using thematic description.

Results and conclusions: Findings suggest that the CLD workshop was effective and feasible in SL practice settings. Data showed that improvement in LPN autonomy and control over decision-making resulted from gaining confidence and feeling empowered, which led to positive change in participants' CL attitudes. Including other team members, health care aides (HCAs), and management in the CLD workshops also improved team relationships for all.
\end{abstract}

Key Words: Clinical leadership, Leadership, Supported living, Nursing, Licensed practical nurses

\section{INTRODUCTION}

The number of older adults is increasing as a result of increased longevity and a decreased birthrate following the post-World War II baby boom. While many older adults maintain good health and independence, advanced age is a risk factor for chronic illness and co-morbid conditions. ${ }^{[1]}$ As a result, older adults are more likely to require additional support with changes in health and lifestyle. In Alberta, Canada, such services may be provided in Supported Living (SL) and Long-Term Care (LTC) settings. In SL, support is provided primarily with activities of daily living and staffing is primarily Licensed Practical Nurses (LPNs) with Registered Nurses
(RNs) predominantly in management roles. ${ }^{[2]}$

The length and content of LPN education programs vary across different provinces in Canada (Table 1). Within Canada, LPN programs vary in length from 16 months (British Columbia - Vancouver Community College) to 30 months (Ontario - Georgian College), with such programs often modified to serve jurisdictional expectations. ${ }^{[3-6]}$ Despite education and licensure variations and requirements, LPNs provide more than $70 \%$ of aged care in North America. ${ }^{[7]}$ They are now responsible for increasingly complex aged care, including supervising care aides, building and leading teams,

${ }^{*}$ Correspondence: Lorraine Venturato; Email: lventura@ ucalgary.ca; Address: University of Calgary, 2500 University Drive NW, Calgary, Alberta, Canada. 
making critical decisions, resolving conflict, dealing with family, and collaborating with other healthcare disciplines. ${ }^{[8]}$ In Alberta, LPNs are authorized to assume administrative and clinical leadership roles in SL, roles which require particular skill sets. Clinical leadership (CL) education and training for LPNs that is work-based and effective offers a potential solution to many of the challenges encountered with increased LPN practice responsibilities. While researchers have investigated CL in nursing, very few have focused on CL with LPNs. It is crucial to study the CL of LPNs separately from that of RNs because LPNs receive different education and training in clinical judgment, critical-thinking skills, and leadership. ${ }^{[3]}$

The growing scope and changing nature of the role of LPNs in SL settings requires an increased focus on the development of new skills, particularly in relation to CL. This thesis study is Phase 2 of a larger multiphase program of research conducted by Dr. Loraine Venturato, Principal Investigator. In Phase 1 of the multiphase program of research, understandings of CL for LPNs in SL in Alberta were qualitatively explored. Findings from Phase 1 suggested that for LPNs in the workplace, there were CL knowledge and skill gaps in communication and teamwork.

Table 1. LPN education programs in Canada

\begin{tabular}{llll}
\hline Province & Program Credential & Academic Institution & Length \\
\hline Newfoundland and Labrador & LPN Diploma & Centre for Nursing Studies & 16 months \\
Nova Scotia & LPN Diploma & Nova Scotia Community College & 2 years \\
New Brunswick & LPN Diploma & New Brunswick Community College & 2 years \\
Manitoba & LPN Diploma & Assiniboine Community College & 2 years \\
Saskatchewan & LPN Diploma & Saskatchewan Polytechnic & 2 years \\
British Colombia & LPN diploma & Vancouver Community College & 16 months \\
Alberta & LPN Diploma & Bow Valley College & 20 months \\
Ontario & LPN Diploma & Georgian College & 30 months \\
Nunavut & LPN Diploma & Nunavut Arctic College & 2 years \\
Quebec & Diploma in Nursing & John Abbott College & 2 years \\
Yukon & LPN Diploma & Yukon College & 2 years \\
Prince Edward Island & LPN Diploma & Holland College & 2 years \\
\hline
\end{tabular}

\subsection{Conceptualizing clinical leadership}

While confusion exists about leadership and clinical leadership terminology, CL is most often defined as informal leadership associated with clinical care and clinical management, undertaken by skilled clinicians without a formal leadership role title. ${ }^{[9]}$ In addition to various definitions of clinical leadership, numerous formal and informal leadership theories circulate, including authentic, transformational, contingency, servant, and congruent clinical leadership theory. ${ }^{[10]}$ Stanley (2008) emphasizes the importance of adopting a leadership theory for $\mathrm{CL}$ that is rigorous and well suited to nursing. ${ }^{[11]}$

Congruent CL theory was developed by David Stanley (2006) as an evidence-informed theory of clinical leadership particular to nursing. ${ }^{[12]}$ This theory highlights nursing values as central to nursing practice and ultimately to leadership (and indeed to followership) in clinical practice. The theory recognizes fundamental nursing values, such as dignity, integrity, trust, autonomy, privacy, altruism, humanity and personhood, hope, security, and social justice. ${ }^{[13,14]}$

Stanley (2017) identified several key components of effec- tive clinical leadership - clinical expertise, communicating effectively, empowering and respecting others, working effectively in a team and building team abilities, driving change, providing quality clinical care, and having a clear vision. ${ }^{[9]}$ Effective clinical leaders engage in role-modelling, education, communication, delegation, value setting, advocacy, teamwork, and act as expert resources. ${ }^{[15]}$

Effective CL has numerous workplace impacts. For example, nursing leadership skills positively influence patient outcomes by helping to ensure patients receive higher quality care. ${ }^{[11,16]}$ The result is decreased rates of infection, pressure ulcers, and behavioural issues among other concerns. ${ }^{[17-19]}$ It also affects employee satisfaction as CL has been linked to increased job satisfaction and improved retention of nurses. ${ }^{[9,20]}$ Venturato and Drew (2010) identify the following outcomes of effective clinical leadership in particular: (a) enhanced staff autonomy and efficiency; (b) increased job satisfaction and reduced staff turnover; and (c) improvement in the quality of care reported by residents, families, and staff. However, despite the many potential benefits of effective CL, little is known about CL among LPNs 
in SL care settings. ${ }^{[15]}$ Notably, despite LPNs' expanding scope of practice and changing roles in SL contexts-so that they often now include leadership — existing research is limited and work-based education to develop LPNs' CL skills is minimal.

\subsection{Developing effective clinical leadership}

In this study, a practice development (PD) approach was used because of its success in transforming the culture of practice. $^{[21]}$ McCormack et al. (2009, p.93) note that the PD purpose is "to develop more effective workplace cultures that have embedded within them person-centered processes, systems and ways of working". [21] Effective facilitation with a PD approach of McCormack, (1998) is based on the assumption that changing behaviours and attitudes depends on enabling participants to become more self-aware; the intention is that they will become motivated and empowered to implement these actions in their everyday work within a culture of constant review and learning. ${ }^{[2,23]}$ Thus, the main purpose of a PD approach is to achieve quality care through developing nurses' knowledge, skills and values-skilled facilitation and continuous, rigorous change enables nurses to transform their work culture. ${ }^{[24]}$

Both congruent CL theory and a PD approach emphasize the relevance of nurses' knowledge, skills, and values to continuously improve and develop nursing practice. As McCormack, Manley, and Titchen (2014) note, effective, efficient, and focused health care requires the use of systematic, facilitative, collaborative, and supportive developments in practice. ${ }^{[25]} \mathrm{A}$ PD approach can help to transform nursing culture to include continuous CL development.

\subsection{Study aims}

This study had three primary aims: to develop and deliver a clinical leadership development (CLD) workshop for LPNs who work in SL settings; to explore the influence of the CLD workshop on LPN clinical leadership (CL) attitudes, knowledge, and skills; and, to explore the feasibility of implementing a work-based CLD workshop.

\subsection{Research questions}

Three research questions directed this study, as follows:

1) What is the LPN experience of the CLD workshop?

2) What influence does a CLD workshop, focused on teamwork and communication, have on the attitudes, knowledge, and skills of LPNs in CL?

3) How feasible is a work-based, CLD workshop for LPNs in an SL setting in Alberta? This exploratory qualitative research study incorporated a demographic questionnaire, individual and focus group interviews to explore changes in CL practice, attitudes, and knowledge for LPNs in SL.

\section{MeTHODS}

This exploratory qualitative research study incorporated a demographic questionnaire, individual and focus group interviews to explore changes in CL practice, attitudes, and knowledge for LPNs in SL.

\subsection{Study setting}

This study was conducted at a SL facility in Alberta, Canada. The study site included 78 supported living suites for older adults. The on-site, 24-hours-a-day care team included LPNs and health care aides (HCAs) with RNs on-call.

\subsection{Participants}

Six LPNs participated in the study. All participants worked in SL and had been employed for a minimum of 20 hours per month over the last six months. All participants were rostered as team leaders on their shifts.

\subsection{Ethics approval}

The study received ethical approval through the University of Calgary Human Research Ethics Board and organizational approval through both the site management and the Research Centre of the larger care organization. All participants provided written informed consent prior to commencing the study.

\subsection{Developing and implementing the clinical leadership development workshop}

The CLD workshop was comprised of three workshop sessions (see Table 2) focused on exploring and addressing current CL challenges LPNs faced in the workplace. The aim of the CLD workshop was to develop LPN CL skills, integrate nursing values into LPN leadership behaviours, and develop LPNs' abilities to apply new CL knowledge and reflections into their practice. Active teaching and learning approaches were incorporated into each of the three sessions. Participants had opportunities to reflect on actual clinical situations, share their thoughts on a current workplace issue, explore how their CL learning might be useful in addressing the issue in future, and plan and implement their action strategies in practice. The researcher provided weekly follow-up visits to support participants, to answer their questions, and to observe their progress. The 1.5-hour CLD workshop sessions were developed and delivered by the researcher over a six-week period. The CLD workshop focused primarily on CL teamwork and communication. The design of the CLD workshop was based on Venturato and Drew's (2010) ${ }^{[15]}$ CL model and Stanley's (2017) ${ }^{[9]}$ congruent CL theory. The CLD workshop included CL content (knowledge) and skill development (application) through the use of interactive and experiential learning in the workplace. 
Table 2. CLD workshop design

\begin{tabular}{|c|}
\hline Session 1 \\
\hline Review: Congruent CL definition \\
\hline Engage in: Values clarification and reflect on current practice (Stanley, 2017) \\
\hline Discuss: What is effective or positive teamwork and communication? \\
\hline Develop: An action plan (focus) for the next ten days \\
\hline Discuss: How do our values translate into becoming a leader? (Stanley, 2017) \\
\hline $\begin{array}{l}\text { Discuss or reflect on: Nursing shared values, such as human dignity, integrity, trust, autonomy, privacy, humanity, hope, security, } \\
\text { personhood, self-sacrifice, and social justice }\end{array}$ \\
\hline Session 2 \\
\hline Reflect on: Their action plan \\
\hline Discuss: Personal and professional values \\
\hline Share and reflect on: Their experience and different style of communication among participants \\
\hline Discuss: An effective communication skill in relation to clinical leadership (feedback, listening, crucial conversations) \\
\hline Discuss and share: Teamwork and communication, and experiences of problem-solving \\
\hline Discuss: Respect, active listening, teamwork, and motivating and empowering other team members \\
\hline Discuss and reflect on: Their experiences of teamwork \\
\hline Develop: An action plan (focus) for the next ten days \\
\hline Discuss: Effective communication, such as feedback, listening, and crucial conversations \\
\hline Discuss: Respect, active listening, teamwork, and motivating and empowering other team members \\
\hline Session 3 \\
\hline Reflect on: Action plan \\
\hline Reflect on: The alignment of participants' nursing and personal values and their actions \\
\hline Discuss: Effective relationships and leadership \\
\hline $\begin{array}{l}\text { Discuss: Effective teamwork attributes such as shared goals, informality, participation, listening, meeting regularity, interdependent } \\
\text { working, civilized disagreement, consensus decisions, clear role, shared leadership, and self-regulations. }\end{array}$ \\
\hline
\end{tabular}

\subsection{Data collection}

Data were collected through focus group and individual interviews held immediately preceding and following the CLD workshop. Two participants were individually interviewed as they were unable to attend the focus groups. An additional individual interview was conducted with the RN Clinical Lead two weeks after the CLD workshop. The primary researcher, completed field notes for each CLD workshop session.

\subsection{Data analysis}

The focus group and individual interview transcripts were thematically analyzed using Braun and Clark's (2006) thematic approach. ${ }^{[2]}$ Thematic analysis involved the search for themes or repeated patterns across entire data sets guided by the study's theoretical framework. The primary researcher, conducted the analysis by extensively reading the transcripts to generate initial themes. The primary researcher, then mapped the themes, which were reviewed, defined, and refined until each theme and sub-theme was named and a detailed description completed for each one.

\section{Results}

Six LPNs participated in this study, all women, the majority (83\%) between 35 to 44 years. Years of nursing experience were evenly split, with 50\% having less than 5 years and 50\% having more than 5 years but less than 10 years (see Table 3).

Table 3. Workshop participant demographics ( $n=6$, all women)

\begin{tabular}{llll}
\hline Demographic Characteristics & & $\mathbf{n}$ & $\mathbf{\%}$ \\
\hline Nursing Background & & 2 & 33 \\
Age group (in years) & $35-44$ & 5 & 83 \\
& $45-54$ & 1 & 17 \\
Hours worked per week & $<30$ & 1 & 17 \\
\multirow{2}{*}{ Work Experience (in years) } & $30+$ & 5 & 83 \\
& $2-5$ & 3 & 50 \\
\hline
\end{tabular}

Note. $\mathrm{N}=6$ and all participants were women.

\subsection{What is the LPN experience of the CLD workshop?}

LPNs' experiences of the CLD workshop are captured in two main themes below.

\subsubsection{Exploring and raising awareness of their role as clinical leader}

Participants demonstrated a shift in awareness from the beginning of the workshop sessions to the end of sessions. Although most participants introduced themselves as a team 
leader prior to the CLD workshop, they saw this title as primarily referring to individual responsibilities for daily tasks: "I am a team leader. I take care of residents."; "I am doing some [of these] tasks." They reported less sense of leadership responsibilities within the team. One LPN expressed: "I was thinking that the leadership just belongs to management. Before [the CLD workshop] I thought it is just a title." During and after the CLD workshop, the participants developed further awareness of their CL roles. They identified their position as a leader within their team and thus advanced towards more understanding of their leadership roles. One participant stated: "We know now that it was our duty and our role to be a problem solver with our team, which was one of the things that you taught us."

Management also recognized a change in participants' understandings of their role as a CL. The clinical manager (CM) noted that: "They learned that they are a leader. That their role is more than just a clinical assessment, medication, et cetera. But they are a leader as well.” During the six weeks of the CLD workshop, the participants were seen to be more responsible, with the CM stating, "What I noticed was they are more willing to work with other team members and solve some of the issues rather than pushing the issue to the next leader in line."

Most of the participants declared that the CLD workshop was an eye-opener for them in relation to their responsibilities as CLs. One participant stated: "To know that a team leader was more than sitting in an office with a job and [the] duty of giving medication and day to day activity. It is way beyond that." Participants noted that the CLD workshop active learning activities allowed them to practise applying their CL learnings and changed the way they saw themselves as leaders in a clinical setting, so that they became more willing to accept responsibilities and to be accountable: "It made us understand that when you are a leader, you have the responsibility of the leadership."

\subsubsection{Sense of empowerment in their role as clinical leader}

Participants initially seemed pessimistic and cynical about the possibilities of CL. They voiced feeling a lack of trust and lack of respect from fellow HCA team members and from management: "We need them [management] to trust us, [and] don't make a decision without discussing it with our team. They felt that no matter what they did or what they learned, it would not lead to any positive change in their practice: "[For] many years we are saying this, but nothing happens yet." Participants also claimed that they didn't have a role in the decisions made on the unit. They considered their lack of confidence as being responsible for their disengagement: "Yes, it was confidence. We didn't have confidence to go because we were afraid that they will turn us down."

Being included in decision making, autonomy, and trust were all important dimensions for the participants. They claimed the development workshops allowed them to take on leadership responsibilities that helped them to solve some of their existing clinical problems and gave them a chance to develop their CL skills. One participant said: "So, instead of pushing the problems to the managers, we can deal with it and try to solve it first. It is good to know and learn how to be a good team leader and also to work with our team." Participants acknowledged that the CLD made them more confidence and empowered them: "I was a little nervous. But after your workshop, I become more confident and I am able to solve more problems about staff related issues." Empowering other team members and motivating and inspiring confidence in the HCAs was another result of the CLD workshop. One participant claimed: "Going and talking to them [management] built our confident. I can apply the same knowledge to motivate my [HCAs] to build up their confidence."

From the management's point of view, the participants started to deal with problems more effectively. The CM stated: "I have seen them try to implement more troubleshooting with their staff and trying to do conflict resolution with staff-which is great." Management noticed that the participants' confidence led to a change in their attitude. The CM mentioned: "The attitude for sure has been changed. They see themselves as a leader rather than just, and I am quoting, '[a]clinical LPN'-but [with] more of a leadership role. I think it really opened their eyes."

\subsection{What influence does a CLD workshop focused on teamwork and communication have on the attitudes, knowledge, and skills of LPNs in clinical leadership?}

The influence of the CLD workshop was expressed in two main themes: CLD workshop influence on teamwork, and influence of CLD workshop on team communication. The CLD workshop influenced on teamwork, focused on relationship, and theme of influence of CLD workshop on team communication, which is further divided into several subthemes of feedback, listening, regular meeting, improved team communication, and conflict resolution.

\subsubsection{CLD workshop influence on teamwork relationship}

Initial observations suggested that staff relationships were quite hierarchical, and teams worked by receiving and giving orders. There appeared to be hesitation with giving and receiving feedback among the team members. Participants expressed feeling uneasy about the possibility that they could 
be reported to management by other team members: "It does affect you. There is no trust or confidentiality." Another participant stated that "they [HCAs] complain behind your back. Rather than sit down and see why the task needs to be done. They didn't listen." Other participants indicated that they felt much the same about this. Participants also viewed the function of their team as being based on who they were working with and their relationship with that individual. One of the participants said: "It [teamwork] can be difficult. It depends on what group you are working with."

Participants reported a change in their relationships within the team after the workshop, due to the change in their approach towards one another. One participant commented: "I found that it brought a good relationship among us." Participants perceived that the CLD workshop helped foster a new relationship among the group because they began to open seek feedback and were also willing to give constructive feedback to their team: "It is like this person trusts me, [when] talking positively about me or giving me positive feedback and then [when] you are telling something negative or something are not working, [then] they look for [a] solution and work hard."

\subsubsection{CLD workshop influence on team communication}

Feedback; Initially, communication between LPNs and HCAs seemed formal and limited. I observed that while HCAs laughed and giggled during the day when passing each other, LPNs remained separate and had their own conversations within their LPN group. Most participants initially classified their communication as a structure of providing reports: "We have a reporting system for that. The RAs report to me and I report to the management." They reported that they received mostly negative feedback (with therefore limited positive feedback) from management: "At least [it would be good] if they would support us, [show some] appreciation and say, 'These guys are doing a good job'.

Listening; During the CLD workshop, participants started to engage in conversations and to listen to the management and HCAs, and to share ideas and opinions: "What I have done since this workshop, at least on the weekend shifts, [it that] we sit and we talk about positive and negative things. And it [has] encouraged staff to be more open with conversations." Dealing positively with communication problems and conflicts was one of the results of the CLD workshop that participants noticed: "In the workshop, we did talk about listening to all part[s] of stories before coming to [a] conclusion."

Regular Meeting; By the end of the CLD workshop, participants were interested in scheduling more regular meetings so that they could improve their communication strategies:
"Us being together, now we know that we are not alone. We understand that this person has the same issue[s]. So, we all can put our head[s] together to see how we are going to solve problems." Also, they declared that meeting one another in the workshops helped them to talk about their problems, share their ideas, and come up with solutions as a team exercise: "We used to have LPN meetings. When we come together as a group of LPNs, we can see the problems."

Improved Team Communication; Participants repeatedly stated that more effective team communication was an outcome of the workshop. They claimed that a constructive feedback approach led to improved effective communication with both management and HCAs. For example, one participant said, "I learned more about communication-how to deal with people, how [to] be more confident, and how to be [a]role model." They believed that they learned effective communication strategies that positively influenced their relationship with management: "You told us how we can communicate to people, how we [can] make others trust us, listen to us, and communicate with them better.". Interestingly, participants perceived that the CLD workshop also made a tangible change in their personal lives by improving their communication skills more generally. One person said, "I use this in my house, which helped me yesterday as well. These skills are not only limited in our workplace, it helps us in our personal life, family life, and relationships."

Conflict Resolution; From a management perspective, the CLD workshop made a noticeable change in participants' communication skills. The CM stated: "Yes, I would say that communication becomes more dialog[ic] in nature. And they realized that it is not going to be a top down observation or top down discussion. It is always going to be, ultimately, 'I have to make decisions,' but they realized that the process is more of the lateral process rather than a vertical process." The CM also noted a change in the way LPNs addressed conflict, commenting that they no longer avoided or shut down when faced with conflict: "[They are] trying to do conflict resolution with staff, which is great."

\subsection{How feasible is clinical leadership education in the workplace, for LPNs in an SL in Alberta?}

Responses were divided into factors that either facilitate or challenge the feasibility of CL education in the workplace as detailed below.

\subsubsection{Facilitators}

Nature of the Workshop; Participants reported that having an interactive and applied workshop made the CLD workshop effective: "[It was] a problem-solving ... workshop [not a teaching one]. You asked what the problem is and what is 
not working, and then you give us strategies to deal with it and how to be a good leader.' In contrast, they shared their ideas about the lack of effectiveness of previous traditional lecturing sessions: "They [other education sessions] come and talk, and we just listen. It goes to here [points to her ear] and goes from here [points to her another ear], but here we brought our own issues, she brought her own, and we learned."

Scheduling; The duration and schedule of the workshops were seen as a part of the success of the workshop, by both management and participants. The CM said, "I think it was a good idea to not just have it [CLD]on one day but have it different weeks... [so] there was essentially follow-up with them [LPNs] and you kept them accountable to do certain tasks by the time you got back here." Scheduling the face-to-face sessions around practical work-based activities encouraged participants to stay engaged and motivated, and to apply their knowledge to their practice: "The reason it felt more [useful was] because we were doing something for the workshop.... Yes, because we were involved. We were motivated."

Management support; This aspect was seen by participants to be critical in gaining initial support for the project, for scheduling workshop sessions, and for engaging the participants (particularly in the initial stages), assigning them tasks, and conducting follow-up. The involvement of the CM was key to motivating participant engagement in practice development and change activities in between workshop sessions.

\subsubsection{Challenges}

Scheduling; Arranging a time for LPNs to attend one and a half hours for the workshop biweekly was complicated because of scheduling and shift coverage needs. Participants also had concerns about the timing of the workshops and wanted to have a choice of the time and the day.

Engagement; I found an initial lack of interest in the study among LPNs during the information sessions. This was where early management support and engagement were critical to ensuring uptake of the workshop. After the first workshop, participants demonstrated more interest and got increasingly involved in the workshop activities. One of the participants explained: "We just want to say that in the beginning we didn't know what we [were] getting, but as the time goes [on], it started to sink in and it sinks [in] more when you take it into our practice."

Sustainability; The CM was concerned about the sustainability of the training for new staff and also the refresher training options. Although both participants and the CM acknowledged the influence of the CLD workshop on LPNs, they were concerned about how they would manage ongoing training: "I thought it was really good-[but] what do we do after that?"

\section{Discussion}

Both participants and management recognized the significant influence of the CLD workshop on LPNs' understandings of their CL roles and CL responsibilities and how they performed their CL role. Thematic analysis findings suggest that developing a CLD workshop for LPNs could increase awareness of CLs, empower LPNs, and improve team communication and teamwork. CLD for LPNs could be effective and feasible in SL. The CLD workshop was effective in that participants felt more confident and empowered, resulting in a positive attitude change towards CL. They expressed improvement in LPN autonomy and in their perceptions of control over decision-making. Participants' attitudes became more positive, which management also noticed. The CLD workshop influenced participants to listen actively, provide and seek constructive feedback, share their opinions, and talk about their concerns and challenges.

The CLD workshop was perceived by participants to have reached its aim of developing CL skills_-in particular, developing effective communication, building relationships, empowering other team members, sharing responsibilities, supporting team-building, and enhancing conflict resolution. Including HCAs in decision-making, seeking their opinions, and being open to their constructive criticism and feedback were key to successfully enhancing teamwork. The results underscore the notion that team-building and communication skills happen in an environment where there is mutual support between all team members. The findings suggest that participants' engagement and interest in the workshop and its activities were related to the workshop focus on participants' actual workplace issues. The workshop was also effective because participants could practise their expanding knowledge and skills, like effective communication strategies and teambuilding, in an active learning group environment.

Venturato and Drew (2010) express the importance of having a "practice orientation" in the context of understanding CL and improving and shifting practice. ${ }^{[15]}$ Stanley and Stanley (2018) also cited Venturato and Drew's study, commenting that the most important benefit of CL education is nurses' expanding understanding about CL and their application of CL to their practice. ${ }^{[10]}$ This study's findings suggest that it is critical that the CLD workshop on CL be offered in the workplace and that the workshop draws on the real life experiences of the LPNs to allow LPNs to learn and to practise, and to critically reflect on CL practice issues that they were experiencing in SL setting. 
In their study, Venturato and Drew (2010) explored the influence of nurses' engagement in CL and the relevance of this aspect to improving the participants' management and communication skills, which resulted in improving nurses' and care workers' retention. ${ }^{[15]}$ This study's findings align this those of Venturato and Drew, which highlighted that when their RN participants were empowered and engaged, they moved from a task-oriented role to a CL role. ${ }^{[15]}$ Stanley (2017) describes "good" or "effective" communication as the main role of a CL. ${ }^{[9]}$ Nurses act in clinician, manager, advocate, or educator roles, which all require effective communication skills. ${ }^{[27]}$ Improving team communication in this study supported the LPNs in their CL role, as they engaged in more active listening, more often provided positive feedback to others, and also sought more feedback.

Stanley (2017) emphasize the importance of team-building and cited Chávez and Yoder (2015) about the necessity of effective team-building skills for CLs. ${ }^{[9,28]}$ The study findings aligned with and demonstrated Stanley's (2017) claim that regular meetings, civilized disagreements, and listening are important attributes of teamwork. ${ }^{[9]}$ Bender (2017) shows that the main attributes of clinical nurse leaders are linked to effective communication and professional relationships, as well as to teamwork and supporting of other staff. ${ }^{[29]}$ Moreover, Antrobus and Kitson (1999) recognized that understanding of self and one's values is an important element in the success of CLs. ${ }^{[30]}$ Similarly, Venturato, Horner, and Etherton-Beer (2019) identify that nurses can improve their practice through critical reflection on their practice, and critical reflection was an ongoing activity of the CLD workshop, focused on improving the LPNs' practice in SL. ${ }^{[31]}$

The study findings suggested that a regularly scheduled CLD workshop offered in the workplace could improve participants' team communication, empowerment, relationships, and teamwork.

\subsection{Recommendations}

A main recommendation from this study is to include HCAs and management in the CLD workshops, to help everyone in the group understand and improve team relationships. Some comments include: "If they come to the workshops and listen to the scenarios, they understand it."; "Maybe the management could be brought in [to the course]."; and "The HCA themselves need to be exposed to something like this. They may not be in a leadership position, but they need to communicate, to give feedback, and to be able to receive feedback."

The care manager also believed that being part of the CLD workshop was very useful, stating, that "I think it was good to get me involved because it really pushes them to have more direct conversations with someone, more in a senior leader position."

\subsection{Limitations and challenges}

This was a small, exploratory study undertaken in one SL site; while other SL sites may have similar staffing models, result are not generalizable.

\section{Conclusion}

Study findings suggest that LPNs working in SL, support and learn from an active learning work-based CLD workshop. The findings reiterate the necessity of work-based CL training to influence LPN CL role-awareness, self-confidence, positive feedback, and conflict resolution. Given the findings of this study, a future research study with a larger sample size is warranted to explore the impact of work-based CLD for LPNs.

\section{ACKNOWLEDGements}

I would like to thank the supportive living facility for providing us with the opportunity to complete this study and for their ongoing collaboration and initiatives. I would also like to express my sincere gratitude to all the participants in the study: The LPNs, education team, and management team. I acknowledge them for generously sharing their time and experiences for the purposes of this study. I remain amazed by all the incredible supported living LPNs who selflessly care for our elderly.

\section{CONFLICTS OF INTEREST Disclosure}

The authors declare that there is no conflict of interest.

\section{REFERENCES}

[1] Grenier É. Canadian seniors now outnumber children for 1st time, 2016 census shows. CBC. Available from: http://wwwcbcca/new s/politics/2016-census-age-gender-14095360

[2] Squires JE, Baumbusch J, Varin MD, et al. A Profile of Regulated Nurses Employed in Canadian Long-Term Care Facilities-CORRIGENDUM. Can J Aging. 2019.
[3] Unruh L. Licensed Nurse Staffing and Adverse Events in Hospitals. Med Care. 2003; 41(1): 142-52. PMid:12544551 https: //doi.org/10.1046/j.1365-2648.1999.00945.x

[4] GC. Practical nursing [iternet].Georgian College (Canada). 2018 July. Available from: https://www.georgiancollege.ca/academi cs/full-time-programs/practical-nursing-pnrs/2018

[5] Butcher DL, MacKinnon KA. Educational silos in nursing education: 
a critical review of practical nurse education in Canada. Nurs Inq 2015; 22(3): 231-9. PMid:25514985 https://doi.org/10.111 $1 /$ nin. 12090

[6] VCC: Nursing Practice. [Internet]. Vancouver: Vancouver Community College (Canada). 2018 June. Available from: http://vcc.ca/programscourses/program-areas/healt $\mathrm{h}$-sciences/practical-nursing/

[7] Corazzini KN, Anderson RA, Mueller C, et al. Licensed practical nurse scope of practice and quality of nursing home care. Nurs Res. 2013; 62(5): 315-24. PMid:23995465 https ://doi.org/10.109 7/NNR.0b013e31829eba00

[8] Tarnowski GJ, Bateman T, Stanger L, et al. Update of Licensed Practical Nurse Competencies in Alberta. Journal of Nursing Regulation 2017; 8(2): 17-22. https://doi.org/10.1016/S2155-8256(17 ) 30094-7

[9] Stanley D. Clinical leadership in nursing and healthcare : values into action: Chichester, West Sussex, England : Wiley-Blackwell Second edition. 2017.

[10] Stanley D, Stanley K. Clinical leadership and nursing explored: A literature search. J Clin Nurs. 2018; 27(9-10): 1730-43. PMid:29076264 https://doi.org/10.1111/jocn.14145

[11] Stanley D. Congruent leadership: values in action. J Nurs Manag. 2008; 16(5): 519-24. PMid:18558922 https ://doi.org/10.111 $1 / j .1365-2834.2008 .00895 . x$

[12] Stanley D. In command of care: toward the theory of congruent leadership...Second of two papers. J Res Nurs. 2006; 11(2): 132-44.

[13] Fagermoen MS. Professional identity: values embedded in meaningful nursing practice. J Adv Nurs. 1997; 25(3): 434-41.

[14] Fahrenwald NL, Bassett SD, Tschetter L, et al. Teaching Core Nursing Values. J Prof Nurs. 2005; 21(1): 46-51. PMid:15682160 https://doi.org/10.1016/j.profnurs.2004.11.001

[15] Venturato L, Drew L. Beyond 'doing': supporting clinical leadership and nursing practice in aged care through innovative models of care. Contemporary Nurse: A Journal for the Australian Nursing Profession. 2010; 35(2): 157-70. https://doi.org/10.1632/prof.2 010.2010 .1 .35

[16] Barba BE, Hu J, Efird J. Quality geriatric care as perceived by nurses in long-term and acute care settings. J Clin Nurs. 2012; 21(5-6): 833-40. PMid:21910775 https://doi.org/10.1111/j.1365-2 $702.2011 .03781 . \mathrm{x}$

[17] Castle NG, Engberg J, Anderson R, et al. Job satisfaction of nurse aides in nursing homes: Intent to leave and turnover. The Gerontologist. 2007; 47(2): 193-204. PMid:17440124 https://doi.org/10 $.1093 /$ geront/47.2.193

[18] McGilton KS, Chu CH, Shaw AC, et al. Outcomes related to effective nurse supervision in long-term care homes: an integrative review. J Nurs Manag. 2016; 24(8): 1007-26. PMid:27573220 https://doi.org/10.1111/jonm.12419

[19] Pearson A, Laschinger H, Porritt K, et al. Comprehensive systematic review of evidence on developing and sustaining nursing leadership that fosters a healthy work environment in healthcare. International Journal of Evidence-Based Healthcare. 2007; 5(2): 208-53. PMid:21631788 https://doi.org/10.1111/j.1479-6988.20 $07.00065 . x$

[20] Curtis EA, Sheerin FK, De Vries J. Developing leadership in nursing: the impact of education and training. Br J Nurs. 2011; 20(6): 344-52. PMid:21471889 https://doi.org/10.12968/bjon.20 11.20 .6 .344

[21] McCormack B, Dewing J, Breslin L, Coyne-Nevin A, Kennedy K, Manning M, et al. Practice development: Realising active learning for sustainable change. Contemp Nurse. 2009; 32(1-2): 92-104.

[22] McCormack B. Primary nursing practice. In primary nursing in Oxford Nursing Development Unit. London: Croom Helm; 1998.

[23] Manley K, O'Keefe H, Jackson C, et al. A shared purpose framework to deliver person-centred, safe and effective care: organisational transformation using practice development methodology. International Practice Development Journal. 2014; 4(1).

[24] McCormack B, Manley K, Garbett R. Practice development in nursing. Oxford, UK Malden, MA: Oxford, UK. Malden, MA: Blackwell Pub.; 2004.

[25] McCormack B, Manley K, Titchen A. Practice Development in Nursing and Healthcare. Hoboken, UNITED KINGDOM: John Wiley \& Sons, Incorporated; 2013. Available from: https://ebookcentr al-proquest-com.ezproxy.lib.ucalgary.ca

[26] Braun V, Clarke V. Using thematic analysis in psychology. Qualitative research in psychology. 2006; 3(2): 77-101. https ://doi.or g/10.1191/1478088706qp063oa

[27] Stavrianopoulos T. The clinical nurse leader. Health Science Journal. 2012; 6(3): 392.

[28] Chávez EC, Yoder LH. Staff Nurse Clinical Leadership: A Concept Analysis. Nurs Forum. 2015; 50(2): 90-100.

[29] Bender M. Conceptualizing clinical nurse leader practice: an interpretive synthesis. J Nurs Manag. 2016; 24(1): E23-E31. PMid:25655928 https://doi.org/10.1111/jonm. 12285

[30] Antrobus S, Kitson A. Nursing leadership: influencing and shaping health policy and nursing practice. J Adv Nurs. 1999; 29(3): 746-53. PMid:10210474 https://doi.org/10.1046/j.1365-2648.19 99.00945. $\mathrm{x}$

[31] Venturato L, Horner B, Etherton-Beer C. Development and evaluation of an organisational culture change intervention in residential aged care facilities. Australas J Ageing. 2020; 39(1): 56-63. 